\title{
Desastres e o ciclo histórico de repetição de tragédias: implicações ao processo de saúde e envelhecimento
}

\author{
Disasters and the historical recurrence of tragedies: \\ implications for the health and aging process
}

Aline Silveira Viana (https://orcid.org/0000-0002-6335-3482) ${ }^{1}$

${ }^{1}$ Centro Nacional de Monitoramento e Alertas de Desastres Naturais. Estrada Dr. Altino Bondensan 500, Eugênio de Melo. 12247-016 São José dos Campos SP Brasil. alinevianagerontologa@ gmail.com

\begin{abstract}
In a context of disaster recurrence, naturalization or demotion of the concept to tragedy does not include all the dynamics and processes involved in affecting the communities and population groups most vulnerable to death and illness, such as the older adults. This paper aims to describe and analyze the socioeconomic, environmental, and health context of impact on older adults in four municipalities in the states of Santa Catarina and Rio de Janeiro. We carried out documentary research and open data survey of sociodemographic, environmental, economic, and health aspects of these locations and population groups. Also, descriptive, interrupted time series and documentary analyses were integrated. Among the results, collected documents and data point to the lack of preventive or mitigating actions for priority groups. Significant changes in hospital admissions for older adults were observed for some groups of causes. We conclude that institutional learning based on collective construction is required to corroborate the disruption of the disaster repetitive cycle.
\end{abstract}

Key words Natural disasters, Older adults, Hospitalization, Socioeconomic aspects
Resumo Em um contexto de recorrência de desastres, a naturalização ou rebaixamento destes à tragédia invisibiliza as dinâmicas e os processos envolvidos na afetação das comunidades e grupos populacionais mais vulneráveis a óbito e adoecimentos, como os idosos. Objetiva-se neste artigo descrever e analisar o contexto socioeconômico, ambiental e de saúde da afetação de idosos em quatro municípios dos estados de Santa Catarina e Rio de Janeiro. Para isto, realizou-se pesquisa documental, levantamento de dados abertos sociodemográficos, ambientais, econômicos e de saúde destas localidades e grupo populacional. Integraram-se as análises descritivas, de série temporal interrompida e documental. Dentre os resultados, observou-se nos documentos e dados levantados carência de previsão de ações preventivas ou mitigatórias voltadas a grupos prioritários. Para alguns grupos de causas, houve mudanças significativas no comportamento das internações hospitalares de idosos no período estudado. Conclui-se que o aprendizado institucional pautado na construção coletiva precisa ocorrer para corroborar com o rompimento do ciclo de repetição de desastres.

Palavras-chave Desastres naturais, Idoso, Internação hospitalar, Aspectos socioeconômicos 


\section{Introdução}

Tragédia é algo que não se espera, nos causa surpresa e demanda organização rápida seja da sociedade ou de órgãos com experiência em responder a eventos ou ocorrências do tipo. Desastres relacionados a eventos ambientais, por sua vez, não poderiam ser compreendidos como tragédias ou ainda como naturais no Brasil, visto o contexto histórico, marcado por seu ciclo de repetição.

Entre os anos 2003 e 2015, por exemplo, em média $25,7 \%$ dos municípios brasileiros decretaram situação de emergência ou estado de calamidade pública' ${ }^{1}$ Trata-se de um dado preocupante, pois os desastres, de forma geral, podem impactar na saúde em tempos variados, desde horas a anos, apresentando efeitos diretos, indiretos e inter-relacionados, cujo cenário de atuação torna-se desafiador aos sistemas de assistência e vigilância em saúde ${ }^{2}$, bem como aos diferentes setores e secretarias que atuam diretamente com a população no nível municipal.

A forma de compreender os desastres, portanto, é fundamental para o delineamento das ações para enfrentar os processos desencadeadores e as expressões destes no tecido social, visto a cronicidade de eventos relacionados que se repetem frequentemente.

Os desastres expressam-se enquanto processo social em um tempo social ${ }^{3,4}$. Na perspectiva da construção social do risco, quatro elementos são imprescindíveis na caracterização de um desastre. Primeiro, os eventos físicos danosos relacionados aos fenômenos meteorológicos, hidrológicos ou climatológicos. Seguem-se, a exposição humana a esses eventos, as condições de vulnerabilidade sociais e ambientais para ocorrência destes e a insuficiência da capacidade local de lidar com os efeitos negativos ou de realizar ações para reduzir os riscos de afetação nesses eventos. ${ }^{5}$.

No cenário nacional, pouco sabemos, todavia, sobre a afetação de idosos e sobre o processo de envelhecimento nesses eventos, cuja complexidade expressa-se em processos interligados de natureza social, ambiental, econômica, cultural, tecnológica, política e física, com rupturas na vida cotidiana que podem chegar à morte biológica ou social ${ }^{4,6}$.

Reconhecer o histórico de desastres e gerar conhecimento/aprendizado a partir dessas experiências é importante, como nos lembram Narváez e colaboradores ${ }^{6}$, pra prevenir riscos futuros, reduzir o risco existente, preparar a resposta, responder, reabilitar, recuperar e reconstruir, dentro de um processo de intervenção que englobe o ris- co e o desastre em diferentes percepções e atores sociais. Com isso, objetiva-se descrever e analisar o contexto socioeconômico, ambiental e de saúde da afetação de idosos em quatro municípios dos estados de Santa Catarina e Rio de Janeiro, severamente afetados por desastres entre os anos de 2008 e 2011.

\section{Métodos}

Os dados e informações coletadas referem-se a quatro municípios brasileiros afetados por desastres, na última década, de grande porte, relacionados a eventos hidrometeorológicos, reconhecidos em âmbito federal: Ilhota/SC, Blumenau/SC, Nova Friburgo/RJ e Teresópolis/RJ. O público estudado é composto por idosos residentes nessas localidades, com idade igual ou superior a 60 anos. Adotou-se o critério para fins de análise, porém como nos lembra Neri ${ }^{7}$, há critérios de classe social, etnia, profissão, educação, outros de origem psicológica e biológica que se entrelaçam a idade para tal caracterização.

Trata-se de uma pesquisa quanti-qualitativa, com emprego de métodos mistos para a coleta e análise dos dados. Compuseram os métodos de coleta de dados, a pesquisa documental e o levantamento de dados secundários em bases de dados, conforme descrito no Quadro 1. Para processamento destas, utilizou-se análise documental, análise estatística descritiva e análise de séries temporais interrompidas da classe ARIMA (autorregressivo integrado de médias móveis), por meio do software Stata ${ }^{\circledR}$ (Quadro 1).

Diferentes elementos do contexto socioeconômico, ambiental e legal, bem como dados de internações hospitalares, de histórico de desastre e de afetação humana nesses cenários são reunidos. Como nos lembra Castellanos ${ }^{8}$, faz-se necessário um esforço para compreensão da saúde enquanto processo. A situação de saúde e suas condições são expressões dos processos de reprodução social, resultante da relação interdependente entre processos complexos, tais como os biológicos, ecológicos, econômicos e reprodutivos das formas de consciência e conduta. Por isso, os elementos incorporados na análise, descritos no Quadro 1, são importantes para a compreensão do retrato encontrado nesses municípios e das possíveis implicações dos desastres à saúde de idosos e aos processos envolvidos no envelhecimento humano. Processos, devido à heterogeneidade do segmento, bem como dos cursos de vida vivenciados por estes ${ }^{7}$. 
Quadro 1. Síntese dos procedimentos metodológicos adotados.

\begin{tabular}{|c|c|c|}
\hline $\begin{array}{l}\text { Objetivos } \\
\text { específicos }\end{array}$ & Fontes utilizadas & $\begin{array}{l}\text { Procedimentos de } \\
\text { coleta e análise }\end{array}$ \\
\hline \multirow[t]{4}{*}{$\begin{array}{l}\text { Descrever } \\
\text { e analisar } \\
\text { o contexto } \\
\text { socioeconômico } \\
\text { e ambiental } \\
\text { de afetação } \\
\text { em desastres } \\
\text { em quatro } \\
\text { municípios } \\
\text { afetados por } \\
\text { desastres em } \\
2008 \text { e } 2011\end{array}$} & $\begin{array}{l}\text { - IBGE Cidades (Censo populacional de 2010) } \\
\text { - Densidade demográfica } \\
\text { - Esperança de vida ao nascer } \\
\text { - Índice de Envelhecimento } \\
\text { - Proporção de domicílios particulares ocupados em } \\
\text { aglomerados subnormais } \\
\text { - Proporção de domicílios em aglomerados subnormais } \\
\text { sem acesso à rede geral de abastecimento de água } \\
\text { - Proporção de domicílios em aglomerados subnormais } \\
\text { sem acesso à rede geral de esgoto ou pluvial } \\
\text { - Proporção de domicílios em áreas urbanas com } \\
\text { ordenamento regular, ligados à rede geral de distribuição } \\
\text { sem bueiro/boca de lobo }\end{array}$ & $\begin{array}{l}\text { Busca de dados referentes aos } \\
\text { municípios estudados } \\
\downarrow \\
\text { Extração de dados } \\
\downarrow \\
\text { Tabulação e interpretação com o } \\
\text { uso do Excel }{ }^{\circledR}\end{array}$ \\
\hline & $\begin{array}{l}\text { Atlas Brasil (com base no censo populacional de 2010) } \\
\text { - IDHM } \\
\text { - Renda per capita (em R\$) } \\
\text { - Proporção de extremamente pobres } \\
\text { - Proporção de pobres } \\
\text { - Índice de Gini }\end{array}$ & $\begin{array}{l}\text { Busca de dados referentes aos } \\
\text { municípios estudados } \\
\downarrow \\
\text { Extração de dados } \\
\downarrow \\
\text { Tabulação e interpretação com o } \\
\text { uso do Excel }^{\circledR}\end{array}$ \\
\hline & $\begin{array}{l}\text {. Sites (para busca dos Planos Diretores dos municípios } \\
\text { estudados) } \\
\text { - Sites oficiais das quatro prefeituras estudadas } \\
\text { - Portal da Legislação } \\
\text { - Diário Oficial }\end{array}$ & $\begin{array}{l}\text { Busca dos planos diretores em } \\
\text { vigor } \\
\downarrow \\
\text { Análise e seleção de artigos sobre } \\
\text { gestão de risco de desastres } \\
\end{array}$ \\
\hline & $\begin{array}{l}\text { S2iD } \\
\text { - Sistema de reconhecimento de situações de emergência } \\
\text { e calamidade pública (jan/1970 a dez/2015) àPortarias, } \\
\text { AVADANs, FIDEs, Jornal, Relatório técnico, } \\
\text { - AVADANs (nov/2008 [SC] e jan/2011 [RJ])à número de } \\
\text { desalojados, desabrigados e afetados em geral com idade } \\
\geq 60 \text { anos }\end{array}$ & \multirow{2}{*}{$\begin{array}{l}\text { Pesquisa documental } \\
\downarrow \\
\text { Levantamento da série histórica } \\
\text { de registros em meses e ano } \\
+ \\
\text { Seleção dos documentos } \\
\text { referente aos desastres estudados } \\
\downarrow \\
\text { Extração de dados de afetação } \\
\downarrow \\
\text { Tabulação e interpretação com o } \\
\text { uso do Excel }{ }^{\circledR}\end{array}$} \\
\hline \multirow{3}{*}{$\begin{array}{l}\text { Analisar o perfil } \\
\text { de morbidade } \\
\text { de idosos } \\
\text { residentes } \\
\text { em quatro } \\
\text { municípios } \\
\text { afetados por } \\
\text { desastres em } \\
2008 \text { e } 2011\end{array}$} & $\begin{array}{l}\text { S2iD } \\
\text { - AVADANs (nov/2008 [SC] e jan/2011 [RJ])à número de } \\
\text { óbitos, enfermos e feridos com idade } \geq 60 \text { anos }\end{array}$ & \\
\hline & $\begin{array}{l}\text { SIH } \\
\text { - Pessoas } \geq 60 \text { anos internadas no SUS no período de } \\
\text { jan/2000 a jan/2018, por local de residência e sexo à taxa } \\
\text { de internação de idosos por 10mil internações ocorridas } \\
\text { no município por todas as faixas etárias e sexo. }\end{array}$ & $\begin{array}{l}\text { Levantamento de dados } \\
\downarrow \\
\text { Tratamento e tabulação dos } \\
\text { dados com o uso do Excel }{ }^{\circledR} \\
\downarrow \\
\text { Análise de séries temporais } \\
\text { interrompidas (ARIMA) com o } \\
\text { uso do Software Stata }{ }^{\circledR}\end{array}$ \\
\hline & $\begin{array}{l}\text {. Rede de assistência (DATASUS) } \\
\text { - Proporção de cobertura populacional estimada para } \\
\text { Estratégia Saúde da Família no mês e ano da decretação } \\
\text { do desastre e em maio de } 2018^{\star}\end{array}$ & $\begin{array}{l}\text { Extração de dados } \\
\downarrow \\
\text { Tabulação e interpretação com o } \\
\text { uso do Excel }{ }^{\circledR}\end{array}$ \\
\hline
\end{tabular}

\footnotetext{
* Mês anterior à coleta dos dados.
}

Legenda: IBGE: Instituto Brasileiro de Geografia e Estatística; S2iD: Sistema Integrado de Informações sobre Desastres da Secretaria Nacional de Proteção e Defesa Civil (SEDEC); AVADAN: Formulário de Avaliação de Danos; FIDE: Formulário de Informações do Desastre; Atlas Brasil: Atlas do Desenvolvimento Humano no Brasil de parceria entre o Programa das Nações Unidas para o Desenvolvimento (PNUD), o Instituto de Pesquisa Econômica Aplicada (IPEA) e a Fundação João Pinheiro (FJP); IDHM: Îndice de Desenvolvimento Humano Municipal; SIH: Sistema de Informações Hospitalares do Sistema Único de Saúde (SUS); Datasus: Departamento de informática do Sistema Único de Saúde do Brasil. 
Para a análise dos dados de internações hospitalares, a princípio todos os capítulos da CID10 foram incluídos. Contudo, pela ausência ou baixa frequência de registros para alguns grupos de doenças, cinco capítulos foram retirados da amostra (Cap. VIII, XV, XVI, XVII e XXI), por não possibilitarem a análise de série temporal na classe ARIMA.

Escolheu-se para a análise de série temporal interrompida, o proposto por Antunes e Cardoso ${ }^{9}$. O mês do desastre na série temporal é demarcado como intervenção (I) e quando esta é significativa (p-value $<0,05$; IC $95 \%$ ), indica impacto imediato (conhecido como degrau ou mudança de nível) e/ou impacto progressivo (conhecido como rampa ou mudança de tendência) nos valores da série temporal.

\section{Resultados e discussões}

\section{Caracterização dos municípios}

Em novembro de 2008, em Santa Catarina, e em janeiro de 2011, no estado do Rio de Janeiro, desastres de grande porte relacionados a deslizamentos e enxurradas afetaram vários municípios, dentre eles destaca-se aqui quatro que apresentaram maior proporção de óbitos e, ao mesmo tempo, características geológicas e climáticas semelhantes, cujos terrenos são acidentados, de alto declive e com rede de drenagem em fundo de vale ${ }^{10}$.

Teresópolis/RJ, Nova Friburgo/RJ e Blumenau/SC são municípios de grande porte (acima de cem mil habitantes). Blumenau/SC, dentre eles, dispõe de maior quantidade e concentração de residentes. Ilhota/SC, de pequeno porte, por sua vez, destaca-se entre estes por apresentar menores índices de desigualdade, pobreza e pobreza extrema (Tabela 1).

Teresópolis/RJ e Nova Friburgo/RJ aproximam-se quanto à densidade demográfica, à esperança de vida ao nascer, ao IDHM e à renda per capita. Teresópolis/RJ, dentre os quatro municípios, revela o cenário mais desfavorável, apontando maior percentil de pobreza, extrema pobreza, domicílios em aglomerados subnormais e sem acesso à rede geral de abastecimento de água, bem como maior índice de desigualdade social, embora acompanhe a média nacional (Tabela 1).

Blumenau/SC, por outro lado, destaca-se entre os comparados por apresentar maior esperança de vida ao nascer e ao mesmo tempo o menor índice de envelhecimento, maior IDHM, maior taxa média anual de crescimento populacional e maior renda per capita. Por outro lado, dispõe de maior proporção de domicílios em aglomerados subnormais sem acesso a rede geral de esgoto ou pluvial (Tabela 1).

\section{Contexto histórico de desastres}

Dada a limitação histórica de falta de registro de desastres, os primeiros registros disponíveis online na Plataforma S2iD do Ministério do Desenvolvimento Regional datam de 1970.

Os principais de registros são de enxurradas $(n=48)$, seguido de tempestades $(n=19)$, inundações $(n=18)$, deslizamentos $(n=8)$, alagamentos $(n=2)$, estiagem $(n=1)$ e incêndio florestal $(n=1)$. Dos municípios, apenas Teresópolis/RJ teve maior quantitativo de deslizamentos.

Referente ao histórico de ocorrências (Figura 1), Blumenau/SC apresenta maior número de registros sobre desastres disponíveis para consulta pública $(n=47)$ e o mais antigo dentre os disponibilizados. Em sequência está Ilhota/SC, com 28 registros, iniciados em 1978; Teresópolis/RJ, com 13 registros, divulgados a partir de 1974; e Nova Friburgo/RJ, com quatro registros disponibilizados a partir de 2005. Contribuem para a explicação do aumento no número de registros, avanços na área de proteção e defesa civil, com ampliação das equipes, equipamentos, sistema de registro e comunicação, criação de leis e dispositivos legais para financiamento das ações ${ }^{11}$.

Os desastres aqui estudados são precedidos de um longo histórico de eventos que antecedem os registros disponibilizados pelas defesas civis na Plataforma S2iD. Como trouxe a pesquisa histórica de Mattedi ${ }^{12}$ sobre a região do vale do Itajaí em SC, há registros de eventos de grande porte nessas localidades datadas da época do Brasil Império. Aqui trabalhamos com os registros oficiais, todavia como evidenciou o estudo de Venturado -Landmann ${ }^{13}$, há desastres de grande porte mesmo na atualidade que não são reconhecidos por meio de decretos.

Visto a recorrência anual de decretações de desastres no país, dos mais diversos portes e tipos, espera-se que haja instrumentos de planejamento e gerenciamento de risco. Entretanto, no ano de 2013, um levantamento chamou a atenção para a situação dos municípios quanto a falta de preparo para situações de emergência e calamidade pública. Neste, 59,4\% dos municípios não tinham nenhum plano de gestão de riscos ${ }^{14}$.

Em Teresópolis/RJ e Ilhota/SC eram ausentes a maioria dos instrumentos elencados, tais 
Tabela 1. Caracterização sociodemográfica de quatro municípios afetados por desastres em 2008 (SC) e 2011 (RJ), com base no Censo de 2010, IBGE.

\begin{tabular}{|c|c|c|c|c|c|}
\hline & $\begin{array}{c}\text { Teresópolis/ } \\
\text { RJ }\end{array}$ & $\begin{array}{c}\text { Nova Friburgo/ } \\
\text { RJ }\end{array}$ & $\begin{array}{c}\text { Blumenau/ } \\
\text { SC }\end{array}$ & $\begin{array}{c}\text { Ilhota/ } \\
\text { SC }\end{array}$ & Brasil \\
\hline \multicolumn{6}{|l|}{ Caracterização do território } \\
\hline População & 163.760 & 182.086 & 304.805 & 12.409 & 190.732 .69 \\
\hline $\begin{array}{l}\text { Densidade demográfica } \\
\left(\mathrm{hab} / \mathrm{km}^{2}\right)\end{array}$ & 210,78 & 194,97 & 591,02 & 48,79 & 22,43 \\
\hline Idosos residentes & $13,2 \%$ & $14,3 \%$ & $9,7 \%$ & $9,6 \%$ & $10,8 \%$ \\
\hline Esperança de vida ao nascer (em anos) & 76,27 & 75,77 & 78,64 & 77,95 & 73,08 \\
\hline Índice de Envelhecimento & $27,5 \%$ & $32,5 \%$ & $23,1 \%$ & $27,4 \%$ & $26,56 \%$ \\
\hline IDHM & 0,730 & 0,745 & 0,806 & 0,738 & 0,727 \\
\hline $\begin{array}{l}\text { Taxa média anual de crescimento } \\
\text { populacional }\end{array}$ & 1,72 & 0,49 & 1,80 & 1,57 & 1,17 \\
\hline $\begin{array}{l}\text { Proporção de domicílios particulares } \\
\text { ocupados em aglomerados subnormais }\end{array}$ & $23,4 \%$ & $0,15 \%$ & $6,8 \%$ & - & $5,62 \%$ \\
\hline $\begin{array}{l}\text { Proporção de domicílios em aglomerados } \\
\text { subnormais sem acesso à rede geral de } \\
\text { abastecimento de água }\end{array}$ & $28,9 \%$ & $0 \%$ & $7,3 \%$ & - & $11,7 \%$ \\
\hline $\begin{array}{l}\text { Proporção de domicílios em aglomerados } \\
\text { subnormais sem acesso a rede geral de } \\
\text { esgoto ou pluvial }\end{array}$ & $55,4 \%$ & $0 \%$ & $60,8 \%$ & - & $32,7 \%$ \\
\hline $\begin{array}{l}\text { Proporção de domicílios em áreas } \\
\text { urbanas com ordenamento regular, } \\
\text { ligados à rede geral de distribuição sem } \\
\text { Bueiro/boca de lobo }\end{array}$ & $18,1 \%$ & $18,6 \%$ & $8,3 \%$ & $50,1 \%$ & $58,5 \%$ \\
\hline \multicolumn{6}{|l|}{ Renda, Pobreza e Desigualdade } \\
\hline Renda per capita (em R\$) & 859,79 & 894,75 & $1.253,17$ & 853,29 & 767,02 \\
\hline Proporção de extremamente pobres & 1,58 & 0,57 & 0,12 & 0,29 & 5,9 \\
\hline Proporção de pobres & 7,06 & 4,08 & 1,02 & 0,97 & 8,5 \\
\hline Índice de Gini & 0,56 & 0,51 & 0,46 & 0,38 & 0,56 \\
\hline
\end{tabular}

como "Leis de uso e ocupação do solo" ou "Plano diretor" que contemplassem a prevenção de enchentes, enxurradas e deslizamentos, bem como "Plano de saneamento básico" para limpeza urbana, manejo de resíduos sólidos e de águas pluviais urbanas ${ }^{14}$. Nenhum dos quatros municípios tinham leis específicas para a prevenção e o enfrentamento de enchentes, enxurradas ou deslizamento, embora recorrentes ${ }^{14}$.

Ao analisar os planos diretores em vigor, entendendo-se estes serem instrumentos primordiais para a gestão municipal direcionar ações de redução de risco de desastres, observa previsão de medidas nesse sentido, contudo, apenas Blumenau/SC teve seu Plano Diretor atualizado após o desastre, com maior detalhamento de ações de prevenção de risco de desastre (RRD).
Em Ilhota/SC, por meio da Lei Complementar no 16/2007, no Art. 20, inciso I, áreas inundáveis na zona rural passam a ser áreas de preservação permanente, juntamente aos topos de morros e nascentes. No Art. 298, a liberação de projeto de parcelamento de solo demanda a análise da presença de zonas com risco de escorregamentos, erosão de grande porte ou inundação ${ }^{15}$.

Em Teresópolis/RJ, na Lei Complementar $n^{\circ}$ 79/2006, no Art. 23, inciso II, o estabelecimento em curto prazo de um Programa de contenção de ocupações irregulares e de remoção de população em áreas de risco. No Art. 61, inciso III, que a ocupação dos terrenos permitam a permeabilidade do solo para redução dos riscos de deslizamentos, assoreamentos e inundações, mas veta edificações em terrenos alagadiços ou sujeitos a 

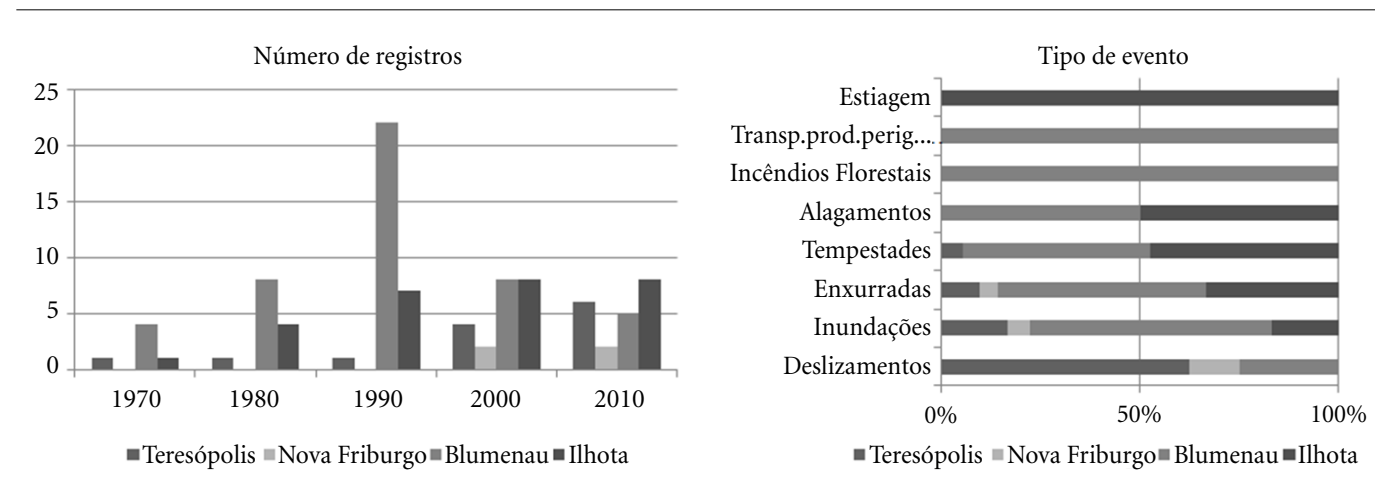

Figura 1. Histórico de situações de emergência e calamidade pública entre 1970 e 2015.

Fonte: S2ID/SEDEC (2018).

inundações. A eliminação de situações de risco e a estabilização de taludes e de margens de córregos são previstas no Art. 110, inciso II, alínea f, bem como a regularização dos assentamentos localizados em áreas de risco, conforme Art. 114 ${ }^{16}$.

Necessário atentar-se, no entanto, se a cartografia do risco está a serviço da população, pois o uso dos mapas técnicos de riscos frequentemente se limita a expulsar moradores de determinadas áreas, sem que o processo decisório seja coletivo, considere diferentes possibilidades de intervenção ${ }^{4,11,17}$.

Blumenau/SC destaca-se por meio da Lei Complementar $n^{\circ}$ 1181/2018, Art. 5, inciso III, por prever a revisão do Plano Diretor mediante ocorrência de desastres que afetem o desenvolvimento urbano, ambiental ou econômico ${ }^{18}$. A Sessão XIII é dedicada à Política Pública Municipal de Prevenção de Desastres, a qual congrega ações de prevenção, mitigação, preparação, resposta e recuperação para evitar ou minimizar os riscos de desastres, com o objetivo do retorno à "normalidade social" 18 .

Necessita-se, no entanto, avanço no debate em defesa civil quanto à "normalidade", permeada pela exposição contínua aos riscos, principalmente em grupos com "mobilidade restrita aos circuitos da vulnerabilidade" ${ }^{\prime 1}$. Esse consentimento/naturalização/incorporação de condições inaceitáveis, injustas ou desiguais é corroborado pela convivência/exposição cotidiana a tais condições e a consequente redução das expectativas para erradicação destas ${ }^{4,17,19}$.

A vulnerabilidade é uma relação e não uma situação de carência a ser suplementada ${ }^{19}$. As ações baseadas na compensação de bens não são suficientes para o enfrentamento e interrupção dos processos e relações que a constitui ${ }^{19}$. Portanto, as ações de enfrentamento precisam ser direcionadas para as causas e as relações geradoras de vulnerabilidade.

Pensando-se no processo de envelhecimento populacional com qualidade, o investimento em ações preventivas integradas, numa abordagem sistêmica, de redução de risco de desastre, beneficiaria não apenas a redução de danos e prejuízos sociais, materiais e econômicos, mas a menor exposição da população a enfermidades, lesões, traumas e risco de óbito. Consequentemente, com ganhos a saúde pública de todos em processo de envelhecimento, não apenas idosos.

Medidas preventivas, como aborda a Política Nacional de Proteção e Defesa Civil ${ }^{20}$, é de responsabilidade não apenas do município, mas dos demais entes federados. Contudo, o orçamento e repasses da união em 2020 para ações preventivas de risco de desastre caíram drasticamente se comparado a anos anteriores. Como traz o levantamento realizado pela Folha de S. Paulo ${ }^{21}$, houve redução progressiva do orçamento para tais ações na última década, sendo que em 2019 apenas um terço do orçamento foi liquidado, e em 2020 o montante disponível correspondeu a apenas 6,7\% do praticado em 2012.

No Plano Diretor em vigor em Nova Friburgo/R ${ }^{22}$, atualmente em revisão, por meio da Lei Complementar no 24/2007, há apenas a previsão no Art. 11, inciso VII, bem como no Art. 27, o reassentamento de populações que ocupam áreas de risco. Contudo, as soluções habitacionais adotadas neste e nos demais municípios estudados, em decorrência dos desastres decretados em nov/2008 (SC) e jan/2011 (RJ) precisariam revistas, por irem contra aos anseios e diretos de mo- 
radia digna aos afetados, em especial à de idosos provenientes de áreas rurais ${ }^{23}$.

Ter instrumentos como o plano diretor é o início de um processo de gerenciamento de risco, desde que utilizado de modo mais participativo e humanizado. O planejamento e gerenciamento voltado para a redução do risco de desastre precisa ser feito junto às comunidades, para que não continuemos a ter grupos repetidamente vulneráveis a tais desfechos, seja eles óbito, desaparecimento, desabrigamento, adoecimento ou até a morte social ${ }^{4}$.

\section{A afetação de idosos nos desastres decretados em nov/2008 e jan/2011}

Em Teresópolis/RJ, 28\% da população reside em áreas de risco de inundações, enxurradas e movimentos de massa. Em Nova Friburgo/RJ, esse percentil é de $18 \%$ e em Blumenau, 25\% ${ }^{24}$. Referente a proporção de idosos residentes em tais áreas ${ }^{25}$, estima-se que em Nova Friburgo/RJ seja de $13 \%$ e, em Teresópolis/RJ, 12\%. Não foram localizados, no entanto, tais dados para Blumenau/SC e Ilhota/SC.

É recomendado cautela na leitura dos dados oficiais reunidos. Em janeiro de 2011, as estatísticas oficiais (Tabela 2) contabilizaram 392 óbitos em Teresópolis/RJ, contudo moradores das áreas afetadas afirmam ser maior o número de mortos, pois os desaparecidos não foram devidamente contabilizados $^{11}$. Além disto, há a indisponibilidade de dados e documentos, como os AVADANs - Avaliação de Danos -, com exceção Blumenau/ SC e Ilhota/SC, detalhados na Tabela 2, os dados dos hospitais de campanha montados para atender aos afetados e os recursos vindos de doação e articulados pelo ente público.

Chama a atenção em Ilhota/SC, os dados de desalojados, deslocados e afetados serem iguais, com os idosos representando $8,6 \%$ do total de afetados de cada tipo. Demonstrando-se uma estimativa dos dados no momento do evento. No caso dos desabrigados, os idosos representaram 18,5\% dos afetados; desaparecidos, 11,1\%; feridos, 6,0\%; enfermos, 39,0\%; e mortos, $3,8 \%$ (Tabela 2).

Em Blumenau/SC, nos dados descriminados por idade, o grupo de idosos compreendeu $12,8 \%$ dos desabrigados, 7,2\% dos feridos e 3,3\% dos enfermos. Observa-se em Ilhota/SC, dessa forma, maior representatividade de idosos desabrigados ( 0,7 vezes maior) e enfermos (11,8 vezes maior) que Blumenau/SC no momento de formulação e preenchimento dos AVADANs (Tabela 2).
E o que acontece com a saúde dos grupos envelhecidos quando ocorre o desastre, no curto e longo prazo? Para isso conduziu-se uma análise de série temporal interrompidas das internações hospitalares no âmbito do Sistema Único de Saúde por todos os grandes grupos de doenças, visando desenhar um retrato macro das principais alterações relacionadas aos desastres estudados (Figura 2).

Era esperado de acordo com as especificidades do desastre, aumento relacionado às causas infecciosas e às causas externas ${ }^{2,26}$, no entanto, outras causas também de desatacaram (Figura 2). No ponto de intervenção na série, em Blumenau/SC, o percentual de mudança anual (APC) para o Intervalo de Confiança (IC) de 95\%, aumentou de modo expressivo nas internações por doenças infecciosas e parasitárias entre idosos do sexo femi-

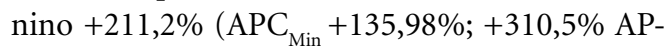
$\mathrm{C}_{\text {Máx }}$; IC 95\%) e masculino $+145,05 \%(+88,65 \%$; $+218,32 \%$; IC 95\%). Em seguida, as internações por doenças do aparelho geniturinário entre idosos do sexo feminino $+32,6 \%(+11,03 \% ;+58,3 \%$; IC $95 \%)$ e masculino $+26,29 \%(+0,47 \% ;+58,8 \%$; IC 95\%) (Figura 2).

Em Teresópolis/RJ, na intervenção da série aumentaram de modo expressivo as internações por doenças dos olhos e anexos para idosos do sexo feminino APC +186,6\% (+27,6\%; +543,6\%; IC 95\%) e masculino APC +87\% (+9,3\%; $+219,9 \%$; IC 95\%). Em seguida, as internações por doenças do sangue e dos órgãos hematopoéticos entre idosas APC +28,21\% (+2,8\%; $+59,9 \%$; IC 95\%); por doenças da pele entre idosas APC $+30,9 \%(+10,9 \%$; $+54,5 \%$; IC 95\%) e idosos $+22,1 \%(+5,98 \%$; $+40,7 \%$; IC 95\%); e por doenças do aparelho geniturinário APC +37,5\% (+11,1\%; +70\%; IC 95\%) (Figura 2).

Em Nova Friburgo/RJ, na intervenção da série destacaram-se as internações por doenças do aparelho digestivo entre idosas APC +93,3\% $(+46,2 \%$; $+155,6 \%$; IC 95\%); por doenças do aparelho geniturinário entre idosos APC $+81 \%$ (+38,6\%; +136,4\%; IC 95\%); por doenças do sangue e dos órgãos hematopoéticos entre idosas APC $+11,54 \%(+3,9 \% ;+19,7 \%$; IC $95 \%)$; e por doenças do aparelho circulatório entre idosas APC +15,8 (+4,5\%; +28,45\%; IC 95\%) (Figura 2).

Em Ilhota/SC há longos períodos com dados iguais a zero na série, comprometendo o método adotado. Como alternativa, retirou-se a variável sexo, agregando-se os dados. Desse modo, sete capítulos da CID-10 trouxeram resultados. Para o ponto de intervenção da série, apenas as internações por sintomas, sinais e achados anormais 
Tabela 2. Distribuição da afetação por recorte etário em quatro municípios.

\begin{tabular}{|c|c|c|c|c|c|c|c|c|}
\hline & \multicolumn{2}{|c|}{$\begin{array}{c}\text { Nova } \\
\text { Friburgo/RJ }\end{array}$} & \multicolumn{2}{|c|}{ Teresópolis/RJ } & \multicolumn{2}{|c|}{ Blumenau/SC } & \multicolumn{2}{|c|}{ Ilhota/SC } \\
\hline & Geral & Idoso & Geral & Idoso & Geral & Idoso & Geral & Idoso \\
\hline Desalojados & 4.528 & -- & 9.110 & -- & 25.000 & -- & 3.500 & 300 \\
\hline Desabrigados & 789 & -- & 6.727 & -- & 5.209 & 667 & 1.300 & 240 \\
\hline Deslocados & -- & -- & -- & -- & -- & -- & 3.500 & 300 \\
\hline Desaparecidos -Avadan & -- & -- & -- & -- & -- & 6 & 18 & 2 \\
\hline Desaparecidos - Minist. Públ. & 274 & 1 & 295 & 7 & -- & -- & -- & -- \\
\hline Levemente feridos & -- & -- & -- & -- & 2311 & 163 & 50 & 3 \\
\hline Gravemente feridos & -- & -- & & -- & 72 & 8 & 17 & 1 \\
\hline Enfermos & -- & -- & -- & -- & 480 & 16 & 41 & 16 \\
\hline Mortos & 429 & -- & 392 & -- & 24 & -- & 26 & 1 \\
\hline Afetados & 5.746 & -- & 16.229 & -- & 103.000 & -- & 3.500 & 300 \\
\hline
\end{tabular}

Fonte: Dados da Proteção e Defesa Civil do Estado do Rio de Janeiro obtidos por Freitas e colaboradores ${ }^{2}$; Avadans da Proteção e Defesa Civil de Santa Catarina disponíveis no S2ID e número de desaparecidos pelo Ministério Público do Rio de Janeiro.

de exames tiveram variação anual de $+60,85 \%$ (Figura 2).

Alguns grupos aqui identificados, como as internações por infecciosas e por causas externas são recorrentes nesses tipos de desastres e entre esse recorte etário ${ }^{2,9}$. Contudo, na literatura poucos estudos sobre os demais grupos de doença foram localizados.

Com resultados que convergem com os aqui observados está o estudo retrospectivo de coor$\mathrm{te}^{27}$ sobre risco de doenças oculares, dermatológicas e gástricas em 22 municípios de Taiwan, afetados por inundações, em um período de 10 anos. Entre as diferentes faixas etárias, os idosos foram os mais suscetíveis aos três tipos de doenças ao longo do período estudado.

Dentre as internações por doenças infecciosas, destacaram-se na maioria dos municípios estudados as por leptospirose e dengue, principalmente nos meses após a intervenção, por ambos os sexos. Estudos nacionais realizados com os demais segmentos etários também vão ao encontro desses achados, como observado por Xavier, Barcellos e Freitas ${ }^{26}$ e Pereira et al..$^{28}$.

Referente às doenças do aparelho geniturinário, um estudo em contexto de terremoto identificou estas como a terceira principal demanda de atendimento médico nos $(10,9 \% \text { dos casos })^{29}$. Pacientes com histórico de problemas renais podem enfrentar dificuldades de acesso a serviços de hemodiálise impactando em hospitalização, como os observados no estudo após a passagem de um furacão, em que $10,3 \%$ de idosos acima de 65 anos perderam três ou mais sessões e 19,8\% perderam uma a duas sessões ${ }^{30}$.
Para as doenças do sangue, não foram localizados estudos sobre idosos nesses contextos. Para o aparelho circulatório, em um estudo ${ }^{31}$ notouse aumento significativo das taxas de hospitalizações por doença cardiovasculares entre idosos, tais como as observadas nesta pesquisa.

Dentre as limitações do método, está a análise por agrupamento das causas de internação por capítulo da CID-10, bem como uso de indicadores sociais, econômicos e ambientais agregados por município e não por setor censitário. $\mathrm{Ou}$ tras pesquisas são necessárias para explorar com profundidade esse tema vasto e complexo, ainda pouco trabalhado no país.

Quando ocorrem os desastres, independente do evento associado - inundação, deslizamentos, entre outros, a afetação dá-se de modo multidimensional ${ }^{4}$, em um tempo social que extrapola o oficialmente reconhecido, envolvendo diversos aspectos da vida da pessoa idosa, como: saúde física e mental, moradia, relações familiares, comunitárias e o ambiente/lugar vivido ${ }^{11}$.

Conhecer a realidade do território, as situações de afetação por desastres e de vulnerabilidade circunscritas neste, a exemplo dos indicadores trabalhados neste artigo, podem auxiliar serviços e programas, seja do setor saúde, assistência social, de proteção e defesa civil, entre outros, para organização de ações de curto, médio e longo prazo em parceria às comunidades.

Importante lembrar que a situação de saúde de idosos nos desastres reflete o contexto no qual estão inseridos, inclusive o de rápido envelhecimento da população se comparado com países europeus, com as mudanças no perfil epidemio- 


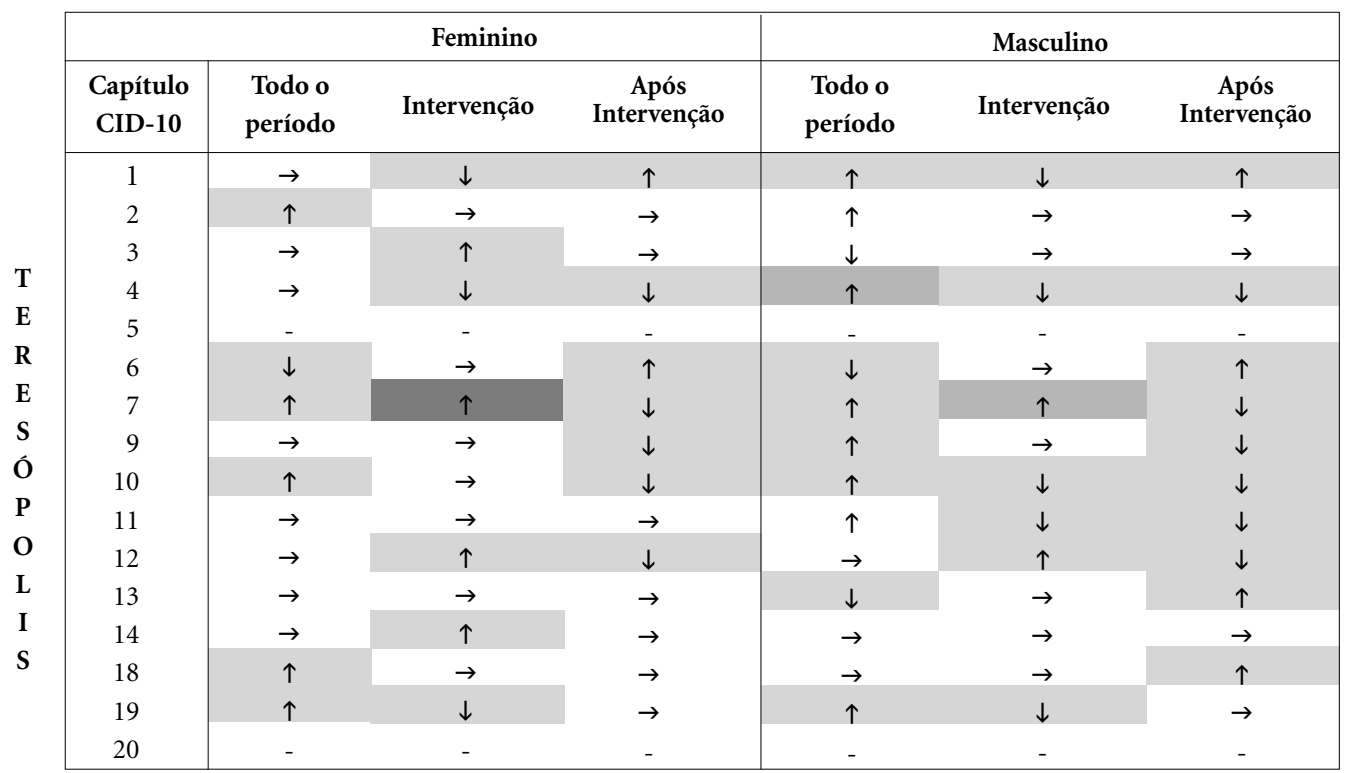

\begin{tabular}{|c|c|c|c|c|c|c|}
\hline \multicolumn{4}{|c|}{ Feminino } & \multicolumn{3}{|c|}{ Masculino } \\
\hline $\begin{array}{l}\text { Capítulo } \\
\text { CID-10 }\end{array}$ & $\begin{array}{c}\text { Todo o } \\
\text { período }\end{array}$ & Intervenção & $\begin{array}{c}\text { Após } \\
\text { Intervenção }\end{array}$ & $\begin{array}{c}\text { Todo o } \\
\text { período }\end{array}$ & Intervenção & $\begin{array}{c}\text { Após } \\
\text { Intervenção }\end{array}$ \\
\hline 1 & $\rightarrow$ & $\rightarrow$ & $\rightarrow$ & $\rightarrow$ & $\downarrow$ & $\uparrow$ \\
\hline 2 & $\uparrow$ & $\downarrow$ & $\rightarrow$ & $\uparrow$ & $\rightarrow$ & $\rightarrow$ \\
\hline 3 & $\rightarrow$ & $\uparrow$ & $\downarrow$ & $\rightarrow$ & $\rightarrow$ & $\rightarrow$ \\
\hline 4 & $\rightarrow$ & $\rightarrow$ & $\downarrow$ & $\rightarrow$ & $\rightarrow$ & $\rightarrow$ \\
\hline 5 & - & - & - & - & - & - \\
\hline 6 & $\rightarrow$ & $\downarrow$ & $\rightarrow$ & $\rightarrow$ & $\downarrow$ & $\uparrow$ \\
\hline 7 & - & - & - & - & - & - \\
\hline 9 & $\rightarrow$ & $\uparrow$ & $\downarrow$ & $\uparrow$ & $\rightarrow$ & $\downarrow$ \\
\hline 10 & $\rightarrow$ & $\rightarrow$ & $\rightarrow$ & $\uparrow$ & $\rightarrow$ & $\rightarrow$ \\
\hline 11 & $\rightarrow$ & $\uparrow$ & $\downarrow$ & $\uparrow$ & $\rightarrow$ & $\downarrow$ \\
\hline 12 & $\rightarrow$ & $\rightarrow$ & $\downarrow$ & $\rightarrow$ & $\rightarrow$ & $\rightarrow$ \\
\hline 13 & $\uparrow$ & $\downarrow$ & $\rightarrow$ & $\rightarrow$ & $\rightarrow$ & $\rightarrow$ \\
\hline 14 & $\rightarrow$ & $\rightarrow$ & $\rightarrow$ & $\rightarrow$ & $\uparrow$ & $\rightarrow$ \\
\hline 18 & - & - & - & - & - & - \\
\hline 19 & $\uparrow$ & $\rightarrow$ & $\rightarrow$ & $\uparrow$ & $\downarrow$ & $\rightarrow$ \\
\hline 20 & $\downarrow$ & $\rightarrow$ & $\rightarrow$ & $\downarrow$ & $\rightarrow$ & $\rightarrow$ \\
\hline
\end{tabular}

Legenda: $\mathrm{p}<0,05=$ significativo.

$\uparrow$ : tendência de aumento. $\downarrow$ : tendência de declínio. Variação de APC \%: $\quad 0-50 \% \quad 50,1-100 \% \square>100,1 \%$

continua

Figura 2. Nível de significância e comportamento da série temporal interrompida de morbidade entre idosos residentes nos municípios (jan/2000-jan/2018).

lógico que tender a ser marcado com enfermidades de longa duração, comorbidades e limitações funcionais e cognitivas ${ }^{8,11}$.

Com o desejo de romper com o ciclo de repetição de tragédias, em um cenário de longevidade populacional crescente, sugerem-se aqui ações integradas de RRD considerando a heterogeneidade dos perfis de envelhecimento e das condições de vulnerabilidade vivenciadas. Indispensável, portanto, envolvimento entre os diferentes 

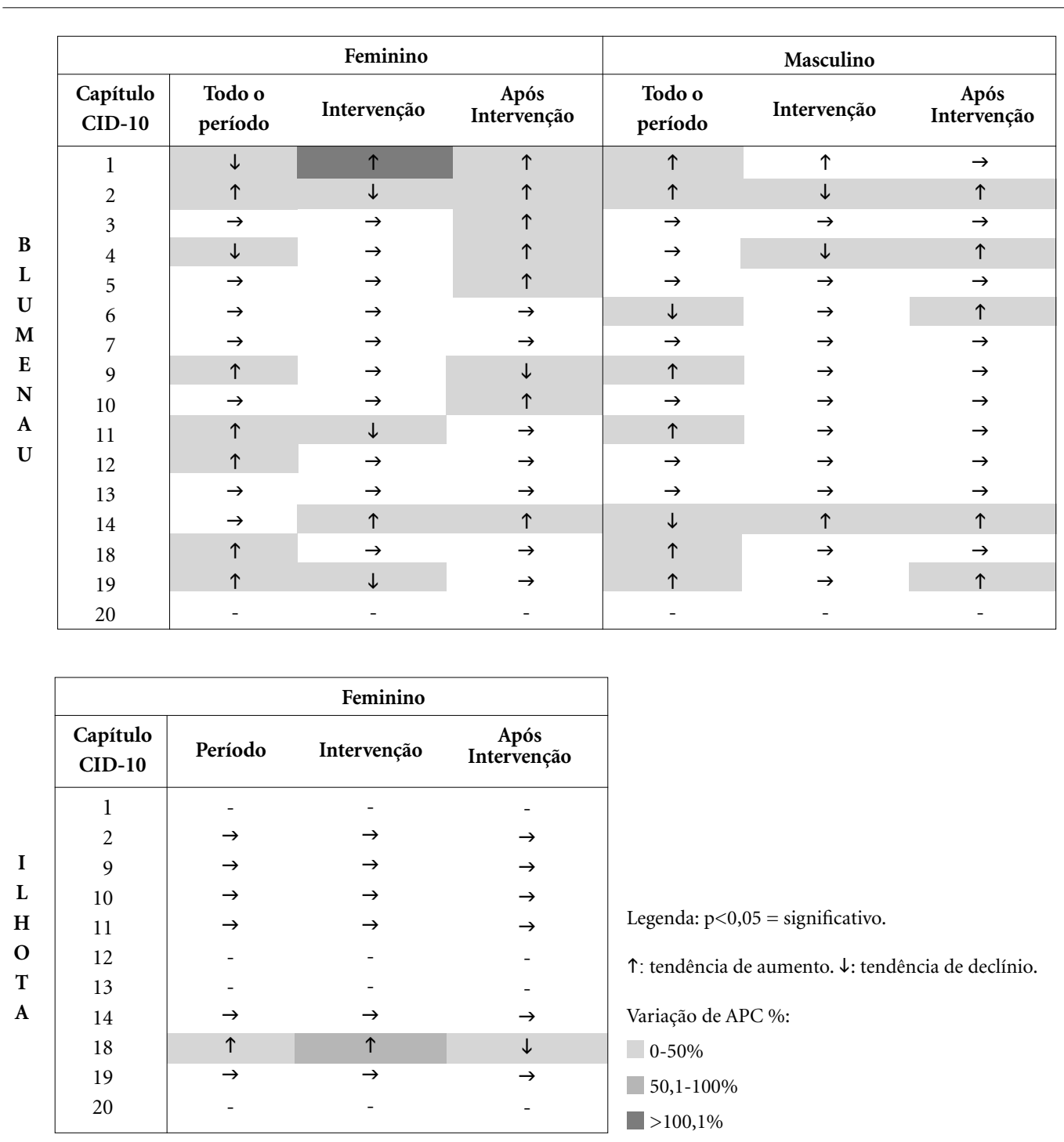

Figura 2. Nível de significância e comportamento da série temporal interrompida de morbidade entre idosos residentes nos municípios (jan/2000-jan/2018).

Fonte: Elaborado pela autora.

atores, como secretarias e órgãos municipais, instituições privadas e não-governamentais, comunidades de áreas consideradas de maior risco de ocorrência de desastres e grupos sociais - tais como idosos, indígenas, mulheres, crianças e pessoas com deficiência física, entre outros.

\section{Considerações finais}

Os municípios estudados têm longo histórico de afetação nos desastres. Levar isso em consideração nos ajuda a entender os desafios que os acompanham ao longo de suas histórias e os reflexos da prioridade ou não de ações sobre o tema no contexto vivenciado.

Não se pode esquecer que neste estudo se abordaram quatro municípios com bom Índice 
de Desenvolvimento Humano Municipal, taxas de Esperança de vida ao nascer e de Renda per capita acima da média nacional e de proporção de pobres abaixo da média nacional. Entretanto, quando ocorre um evento, se não há planejamento conjunto efetivo com base no conhecimento local, pode-se ter consequências como a repetição de desastres e uma atuação institucional pautada em tragédias anunciadas.

$\mathrm{O}$ aprendizado institucional é fundamental para uma melhor prestação de serviços, para mudança no paradigma de atuação centrado na resposta e para romper com a produção de situações que perpetuam os desastres como tragédias recorrentes. O conhecimento gerado precisa darse de modo compartilhado com as comunidades, com as diferentes secretarias e com os setores da sociedade. Ter um cenário em que os desastres se perpetuam como parte da crise crônica instalada no tecido social é inaceitável, pois incorre em consequências a sobrevivência, saúde e bem-estar à população exposta aos riscos.

\section{Agradecimentos}

Gostaria de agradecer o apoio financeiro do Conselho Nacional de Desenvolvimento Científico e Tecnológico (CNPq) ao longo do processo de doutoramento, do qual deriva o artigo, assim como a orientação do Prof. Dr. Carlos Machado de Freitas.

\section{Referências}

1. Brasil. Ministério da Integração Nacional (MI). Reconhecimentos Realizados e Reconhecimentos Vigentes. [internet]. [acessado 2019 set 25]. Brasília: MI; 2018. Disponível em: http://mi.gov.br/web/guest/reconhecimentos-realizados

2. Freitas CM, Silva DRX, Sena ARM, Silva EL, Sales, LBF, Carvalho ML, Mazoto ML, Barcellos C, Costa AM, Oliveira MLC, Corvalán C. Desastres naturais e saúde: uma análise da situação do Brasil. Cien Saude Colet 2014; 19(9):3645-3656.

3. Quarantelli EL. What is a disaster? Perspectives on the question. London, New York: Routledge; 1998.

4. Valencio NFLS. Para Além do 'Dia do Desastre'. Curitiba: Editora Appris; 2012.

5. Oliver-Smith A. Global changes and the definition of disaster. In: Quarantelli EL, organizador. What is a disaster? Perspectives on the question. London, New York: Routledge; 1998. p.177-194.

6. Narváez L, Lavell A, Ortega GP. La gestión del riesgo de desastres: un enfoque basado en procesos. San Isidro: Secretaría General de la Comunidad Andina. Peru; 2009.

7. Castellanos PL. Sistemas nacionales de vigilância de la situacion de salud segun condiciones de vida y del impacto de las acciones de salud y bienestar. Programa Análisis de la Situación de Salud y Sus Tendencias. Organización Panamericana de la Salud/OMS; 1991.

8. Neri AL, organizador. Desenvolvimento e Envelhecimento: perspectivas biológicas, psicológicas e sociológicas. 3a ed. Campinas: Papirus; 2001; 2007.

9. Antunes JLF, Cardoso MRA. Uso da análise de séries temporais em estudos epidemiológicos. Epidemiol Serv Saude 2015; 24(3):565-576.

10. Universidade Federal de Santa Catarina (UFSC). Centro Universitário de Estudos e Pesquisas sobre Desastres. Atlas brasileiro de desastres naturais 1991 a 2012: volume Rio de Janeiro e volume Santa Catarina. Florianópolis: CEPED UFSC; 2013. 
11. Viana AS. O Desastre e o Caos Velado: o enfrentamento individual, interpessoal e coletivo de idosos e familiares. $2^{\text {a }}$ ed. Newcastle: Red Waterlat, EDUEPB, CICCUS, IELAT; 2020.

12. Mattedi MA. As enchentes como tragédias anunciadas: impactos da problemática ambiental nas situações de emergência em Santa Catarina [tese]. Campinas: Universidade de Campinas; 1999.

13. Venturato-Landmann R. "A Alagação Ofende": A invisibilidade de um desastre relacionado às cheias atípicas na RESEX Alto Juruá, Acre [tese] São Carlos: Universidade Federal de São Carlos; 2014.

14. Instituto Brasileiro de Geografia e Estatística (IBGE). Perfil dos Municípios Brasileiros; 2013. [acessado 2019 out 10]. Disponível em: https://ww2.ibge.gov.br/munic_social_2013/ver_tema.php?tema=t9_2\&muni$\mathrm{c}=330580$ \&uf $=33$ \&nome $=$ teres $\% \mathrm{C} 3 \% \mathrm{~B} 3$ polis

15. Prefeitura Municipal de Ilhota. Lei Complementar no $16 / 2007$. Dispõe sobre o Plano Diretor e dá outras providências. Versão compilada de 16/11/2017. Diário Oficial da União; 2007.

16. Prefeitura Municipal de Teresópolis. Lei Complemen$\operatorname{tar} n^{\circ} 79 / 2006$. Dispõe sobre o Plano Diretor e dá outras providências. Diário Oficial da União; 2006.

17. Siena M. A atenção social nos desastres: uma análise sociológica das diversas concepções de atendimento aos grupos sociais afetados [tese]. São Carlos: Universidade Federal de São Carlos; 2013.

18. Prefeitura Municipal Blumenau. Lei Complementar $n^{\circ} 1181 / 2018$. Dispõe sobre o Plano Diretor e dá outras providências. Diário Oficial da União; 2018.

19. Acselrad H. Vulnerabilidade Ambiental, processos e relações. Comunicação ao II Encontro Nacional de Produtores e Usuários de Informações Sociais, Econômicas e Territoriais. Rio de Janeiro: FIBGE; 2006.

20. Brasil. Lei 12.608/2012. Dispõe sobre a Política Nacional de Proteção e Defesa Civil e dá outras providências. Diário Oficial da União; 2012.

21. Folha de S. Paulo. Gasto do governo federal com prevenção de desastres é o menor em 11 anos. [internet] 2020. [acessado 2020 nov 19]. Disponível em: https:// wwwl.folha.uol.com.br/cotidiano/2020/01/gasto-dogoverno-com-prevencao-de-desastres-e-o-menorem-11-anos.shtml

22. Prefeitura Municipal de Nova Friburgo. Lei Complementar No 24/2007. Dispõe sobre o Plano Diretor e dá outras providências. Diário Oficial da União; 2007.

23. Viana AS. La vivienda ante desastres: un análisis de las respuestas institucionales años después de la emergência. REDER 2021; 5(2) (no prelo).

24. Instituto Brasileiro de Geografia e Estatística (IBGE). População em áreas de risco no Brasil. $1^{\mathrm{a}} \mathrm{ed}$. Rio de Janeiro: IBGE; 2018.
25. Dias MCA, Saito SM, Alvalá RCS, Stenner C, Pinho G, Nobre CA, Fonseca MRS, Santos C, Amadeu P, Silva D, Lima CO, Ribeiro J, Nascimento F, Corrêa CO. Estimation of exposed population to landslides and floods risk areas in Brazil, on an intra-urban scale. Int J Disaster Risk Reduct 2018; 31(1):449-459.

26. Xavier DR, Barcellos C, Freitas CM. Eventos climáticos extremos e consequências sobre a saúde: o desastre de 2008 em Santa Catarina segundo diferentes fontes de informação. Ambient Soc 2014; 17(4):273-294.

27. Huang LY, Wang YC, Wu CC, Chen YC, Huang YL. Risk of Flood-Related Diseases of Eyes, Skin and Gastrointestinal Tract in Taiwan: A Retrospective Cohort Study. PLoS ONE 2016; 11(5):e0155166.

28. Pereira CAR, Barata MML, Hoelz MPC, Medeiros VNLO, Marincola FCV, Neto CC, Marinho DP, Oliveira TVS, Trigo AGM, Medeiros TK. Avaliação econômica dos casos de Dengue atribuídos ao desastre de 2011 em Nova Friburgo (RJ), Brasil, Rio de Janeiro. Cien Saude Colet 2014; 19(9):3693-3704.

29. Firoozabadi MHD, Abedinzadeh, M, Moslemi, MK. Genitourinary system trauma after 2003 Bam earthquake in Kerman, Iran. Therapeutics and Clinical Risk Management 2011; 7:49-52.

30. Anderson AH, Cohen AJ, Kutner NG, Kopp JB, Kimmel PL, Muntner P. Missed dialysis sessions and hospitalization in hemodialysis patients after Hurricane Katrina. Kidney Int; 2009, 75(11):1202-1208.

31. Becquart NA, Naumova EN, Singh G, Chui K. Cardiovascular Disease Hospitalizations in Louisiana Parishes' Elderly before, during and after Hurricane Katrina. Int J Environ Res Public Health 2018; 16(1):74.

Artigo apresentado em 20/11/2020

Aprovado em 24/05/2021

Versão final apresentada em 26/05/2021

Editores-chefes: Romeu Gomes, Antônio Augusto Moura da Silva 